\title{
Challenges in the Treatment of Newly Diagnosed and Recurrent Primary Central Nervous System Lymphoma
}

\author{
Matthias Holdhoff, MD, PhD'; Maciej M. Mrugala, MD, PhD²; Christian Grommes, MD; Thomas J. Kaley, MD;
} Lode J. Swinnen, MD ${ }^{1}$; Carlos Perez-Heydrich, BS ${ }^{1}$; and Lakshmi Nayak, MD ${ }^{4}$

\section{ABSTRACT}

Primary central nervous system lymphomas (PCNSLs) are rare cancers of the central nervous system (CNS) and are predominantly diffuse large B-cell lymphomas of the activated B-cell (ABC) subtype. They typically present in the sixth and seventh decade of life, with the highest incidence among patients aged $>75$ years. Although many different regimens have demonstrated efficacy in newly diagnosed and relapsed or refractory PCNSL, there have been few randomized prospective trials, and most recommendations and treatment decisions are based on single-arm phase II trials or even retrospective studies. High-dose methotrexate (HD-MTX; $3-8 \mathrm{~g} / \mathrm{m}^{2}$ ) is the backbone of preferred standard induction regimens. Various effective regimens with different toxicity profiles can be considered that combine other chemotherapies and/or rituximab with HD-MTX, but there is currently no consensus for a single preferred regimen. There is controversy about the role of various consolidation therapies for patients who respond to HD-MTX-based induction therapy. For patients with relapsed or refractory PCNSL who previously experienced response to HD-MTX, repeat treatment with HD-MTX-based therapy can be considered depending on the timing of recurrence. Other more novel and less toxic regimens have been developed that show efficacy in recurrent disease, including ibrutinib, or lenalidomide \pm rituximab. There is uniform agreement to delay or avoid whole-brain radiation therapy due to concerns for significant neurotoxicity if a reasonable systemic treatment option exists. This article aims to provide a clinically practical approach to PCNSL, including special considerations for older patients and those with impaired renal function. The benefits and risks of HD-MTX or highdose chemotherapy with autologous stem cell transplantation versus other, better tolerated strategies are also discussed. In all settings, the preferred treatment is always enrollment in a clinical trial if one is available.

J Natl Compr Canc Netw 2020;18(11):1571-1578 doi: $10.6004 /$ jnccn.2020.7667

${ }^{1}$ The Sidney Kimmel Comprehensive Cancer Center at Johns Hopkins,

Baltimore, Maryland; ' ${ }^{2}$ epertment of Neurology, Mayo Clinic, Phoenix, Arizona; ${ }^{3}$ Department of Neurology, Memorial Sloan Kettering Cancer Center, New York, New York; and ${ }^{4}$ Center for CNS Lymphoma, Dana-Farber Cancer Institute, Boston, Massachusetts.
Primary central nervous system lymphomas (PCNSLs) are predominantly diffuse large B-cell lymphomas (DLBCLs) that involve the brain, eyes, leptomeninges, or spinal cord without evidence of systemic involvement. Molecular and gene expression profiling studies demonstrate that most PCNSLs are of the activated B-cell type of DLBCL and often carry mutations in MYD88 $8^{L 265 P}$ and $C D 79 B .^{1-5}$ With an estimated incidence of 1,500 cases per year in the United States, they account for $6 \%$ of all newly diagnosed primary malignancies in the CNS. The incidence of PCNSL in immunocompetent patients has been increasing, with the highest increase in those of advanced age. ${ }^{6-8}$

Many different regimens, predominantly with a highdose methotrexate (HD-MTX) backbone, have demonstrated efficacy in the treatment of newly diagnosed and relapsed or refractory $(\mathrm{R} / \mathrm{R})$ PCNSL, but currently there is no consensus on the optimal treatment of these patients. Very few prospective randomized controlled studies have been conducted, and most recommendations and decisions are based on single-arm phase II trials or retrospective studies. This is in part due to the rarity of this disease, as well as different personal and institutional experiences favoring one specific HD-MTX regimen. More aggressive, potentially more effective therapies have been investigated that include high-dose chemotherapy (HDC) with or without autologous stem cell transplant (ASCT); however, these regimens are limited to fitter, and often younger, patients. Treatment options that are more suitable for elderly or frail patients exist, and there is increasing evidence that these treatments can be effective. ${ }^{9}$

This review aims to provide a structured overview of currently available, evidence-based approaches for the treatment of newly diagnosed and R/R PCNSL. Treatment options are discussed in relationship to the eligibility of patients to tolerate the respective regimens. The 3 main eligibility groups, both for newly diagnosed and R/R PCNSL, used in this review are patients who are (1) candidates for HD-MTX-based treatment followed by HDC and ASCT; (2) candidates for HD-MTX-based therapy, but not for HDC and ASCT; and (3) not suitable 
for HD-MTX or other more aggressive treatments. The predominant theme throughout this discussion is the lack of consensus regarding a best regimen in most settings, and we strongly urge clinicians to consider clinical trials for these patients if one is available.

\section{Treatment of Newly Diagnosed PCNSL}

The formal diagnosis of PCNSL requires comprehensive workup and staging. In addition to imaging of the brain and entire spine using MRI with contrast, cerebrospinal fluid analysis (CSF) and ophthalmologic examination to rule out ocular involvement are necessary. Brain biopsy is typically required to make the diagnosis of lymphoma unless CSF or vitreoretinal cytopathology and flow cytometry are diagnostic. In addition to CNS staging as described earlier, patients with confirmed diagnosis of PCNSL should undergo systemic staging to rule out systemic lymphoma. Although clinical practices vary, most institutions would recommend CT of the chest, abdomen, and pelvis; body PET/CT; testicular ultrasound in older men (aged $>65$ years); and, in rare situations, bone marrow biopsy. Serologic studies include testing for HIV, because PCNSL in immunosuppressed patients requires specific considerations and initiation of antiretroviral therapy, as well as for hepatitis B and C virus, because these may be reactivated in the context of therapy (Figure 1). ${ }^{10}$

Once the diagnosis is confirmed, treatment must begin expeditiously because PCNSL can progress rapidly. HD-MTX-based therapies (MTX dose of 3-8 $\mathrm{g} / \mathrm{m}^{2}$ ) are the standard for induction therapy in patients considered able to tolerate this therapy. Although age and performance status are known prognostic factors and are considered when deciding treatment, the key criterion for distinguishing patients who can tolerate high-dose MTX from those who cannot is renal function; patients with creatinine clearance of $\geq 40 \mathrm{~mL} / \mathrm{min}$ should be considered for treatment with HD-MTX-based therapy. However, appropriate dose reductions must be applied. ${ }^{11,12}$ It should be emphasized that even some patients with poor performance status at diagnosis, if this status is due to the CNS lymphoma, can dramatically improve with HD-MTX-based therapy and achieve long-term remissions. Similarly, elderly patients also may benefit substantially from HD-MTX-based therapy and should not be excluded from treatment solely on the basis of age. Patients experiencing MTX-associated renal failure may be reconsidered for treatment with HD-MTX if their renal function recovers. At that time, using a lower dose of MTX should be considered before returning to full dosing.

There are institutional differences regarding the choice of additional antineoplastic medications to combine with HD-MTX, and patient comorbidities may guide the clinician to select the most suitable regimen for an individual patient. Frequently used additions to HD-MTX include rituximab, procarbazine, and vincristine, and rituximab and temozolomide (Table 1). Based on available literature and evidence, no one regimen is clearly the most effective. Some of the more aggressive regimens that include polychemotherapy, HDC, or HDC/ASCT as consolidation have shown favorable results. It must be acknowledged that these studies typically included younger and healthier patients, and that more frail, elderly patients would not have been included in these comparatively promising datasets. The most common

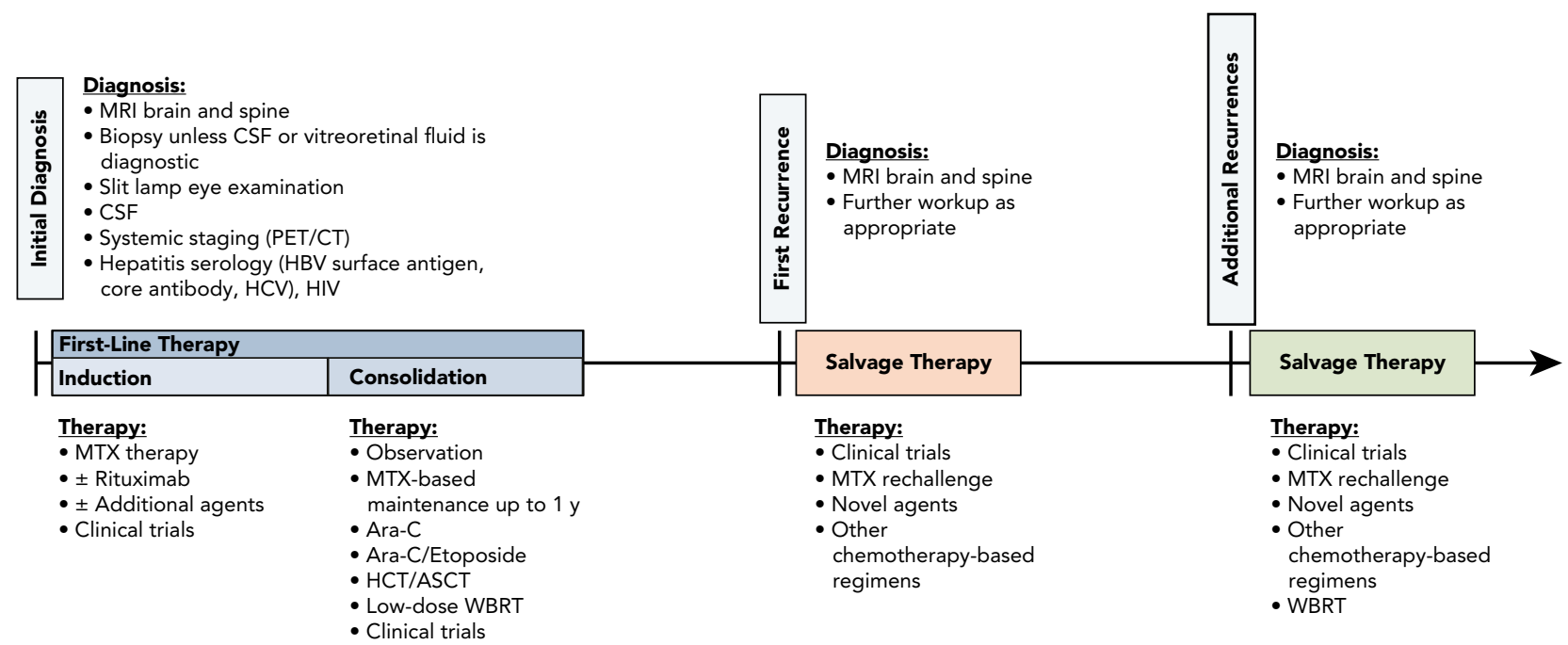

Figure 1. Overview of diagnostic workup and treatment considerations for patients with primary central nervous system lymphoma at time of initial diagnosis, first recurrence, and additional recurrences.

Abbreviations: Ara-C, cytarabine; CSF, cerebrospinal fluid; HBV, hepatitis B virus; HCT/ASCT, high-dose chemotherapy and autologous stem cell transplant; HCV, hepatitis $C$ virus; MTX, methotrexate; WBRT, whole-brain radiation therapy. 
Table 1. Possible Treatment Regimens for Patients With Newly Diagnosed PCNSL

\begin{tabular}{|c|c|c|c|c|}
\hline \multicolumn{3}{|c|}{$\begin{array}{l}\text { Strategies With Consolidation } \\
\text { for Fit Patients }\end{array}$} & \multicolumn{2}{|c|}{$\begin{array}{l}\text { Strategies Without Consolidation, } \\
\text { Including for Elderly or Frail Patients }\end{array}$} \\
\hline Induction & Consolidation & References & Induction/Maintenance & References \\
\hline MTX, TMZ, rituximab & Low-dose WBRT + TMZ & Glass et al, $2016^{50}$ & $\begin{array}{l}\text { Rituximab, MTX (if can } \\
\text { tolerate), PCZ, lomustine }\end{array}$ & Fritsch et al, $2011^{51}$ \\
\hline $\begin{array}{l}\text { MTX, Ara-C, thiotepa, } \\
\text { rituximab }\end{array}$ & WBRT or HDC/ASCT & Ferreri et al, $2016^{53}$ & TMZ + rituximab & Nayak et al, $2013^{15}$ \\
\hline MTX, PCZ, VCR, rituximab & HDC/ASCT (age $<65$ y) & Omuro et al, $2015^{25}$ & TMZ & Kurzwelly et al, $2010^{16}$ \\
\hline $\begin{array}{l}\text { MTX, carmustine, teniposide, } \\
\text { prednisone } \pm \text { rituximab }\end{array}$ & $\begin{array}{l}\text { HiDAC (+ low-dose WBRT } \\
\text { in patients aged } \leq 60 \mathrm{y} \text { ) }\end{array}$ & Bromberg et al, $2019^{54}$ & & \\
\hline
\end{tabular}

Abbreviations: Ara-C, cytarabine; ASCT, autologous stem cell transplantation; HDC, high-dose chemotherapy; HiDAC, high-dose cytarabine; MTX, methotrexate; PCNSL, primary central nervous system lymphoma; PCZ, procarbazine; TMZ, temozolomide; VCR, vincristine; VP-16, etoposide; WBRT, whole-brain radiation therapy.

criterion for HDC/ASCT eligibility is age, although there is no absolute cutoff. Generally, patients aged $<60$ years are more often considered for this modality; however, those aged 60 to 70 years can also benefit. ${ }^{13}$

For patients who are not eligible for HD-MTX, there is no clear best standard treatment, and selection of the most appropriate regimen should at least in part be based on patient comorbidities and physician expertise. Often referral to a center with expertise in the management of PCNSL should be considered. Regimens considered in this setting include temozolomide and rituximab, often used in combination, ${ }^{14-16}$ or other agents typically reserved for treatment of recurrence tried in the first line in this patient population. For example, ibrutinib and lenalidomide have demonstrated efficacy in the recurrent setting, ${ }^{17}$ but can be considered as first-line treatment in patients for whom HD-MTX is not feasible. Moreover, clinical trials using novel agents may allow enrollment of patients with newly diagnosed PCNSL who are too frail for conventional MTX chemotherapy.

Whole-brain radiation therapy (WBRT) offers high response rates in PCNSL, but unfortunately the responses are not durable. Additionally, the significant neurotoxicity of WBRT at the doses administered for upfront therapy makes this modality unattractive for older patients. Therefore, WBRT is not recommended as first-line treatment in newly diagnosed patients.

\section{Role of Consolidation Therapy in Newly Diagnosed PCNSL}

Multiple consolidation therapies are in use today, but similar to the induction regimens described earlier, no clear consensus exists on the optimal choice for consolidation. In addition, the question of the added value of consolidation versus observation after remission has never been conclusively addressed in prospective clinical trials. The main goal of consolidation is to significantly improve progression-free survival (PFS) and delay relapses. The 3 main modalities used for consolidation in PCNSL include nonmyeloablative chemotherapy, HDC/ASCT, and WBRT. Historically, WBRT was commonly used as an effective method of consolidation following chemotherapy-based induction regimens. ${ }^{18,19}$ Although WBRT can generate rapid radiographic responses, they are not always durable. In addition, radiographic response assessment can be affected when WBRT is added after systemic induction. Moreover, significant neurotoxicity and lack of coverage for CSF-disseminated disease make full-dose RT less than ideal. Although combined treatment modalities using HD-MTX followed by WBRT at a reduced $\operatorname{dose}^{20}$ may mitigate the neurotoxicity, newer approaches such as dose-intensive chemotherapy and HDC/ASCT have emerged as viable options.

Nonmyeloablative chemotherapy strategies have been studied and may offer a reasonable alternative to WBRT or ASCT in some patients, although again, no consensus exists on the best approach. The value of consolidative high-dose cytarabine (HiDAC) after HDMTX has been investigated in a prospective phase II study in elderly patients (age $>60$ years), ${ }^{21}$ and a PFS advantage has been demonstrated for consolidative HiDAC after HD-MTX in a randomized phase II trial. ${ }^{22}$ Additionally, intensified conventional chemotherapy was studied in a prospective ALLIANCE phase II trial. ${ }^{23}$

Several studies investigating HDC followed by ASCT as part of initial therapy have demonstrated high complete response and 2-year PFS rates. ${ }^{24,25}$ Currently 2 clinical trials are investigating whether there are significant differences in outcomes between HDC followed by ASCT and consolidative conventional-dose chemotherapy (NCT01511562). ${ }^{26}$ Trials examining consolidative conventional-dose chemotherapy and consolidative 
full-dose WBRT have shown similar 2-year PFS in randomized phase II trials; however, the toxicity profiles of these therapies differ, and longitudinal neurocognitive assessment showed persistent and significant neurocognitive impairment in the patients who received consolidative WBRT but not in the HDC group. ${ }^{13,27}$ Reduced-dose WBRT has recently been shown in a randomized study to improve PFS (ClinicalTrials.gov identifier: NCT01399372), although long-term neurocognitive studies are pending. It is important to note that patient selection in the HDC trials may have played a role in these favorable outcomes.

\section{Treatment of R/R PCNSL}

More than 25 different regimens have been published for patients with R/R PCNSL in several prospective, but mostly retrospective, reports. ${ }^{28}$ However, no prospective randomized trials have been published in this setting. Patients with refractory disease or early recurrence need to be differentiated from those who had remained in prolonged remission after initial HD-MTX-based therapy. What defines a meaningful minimum time in remission to consider repeat treatment with HD-MTX remains controversial. The NCCN Clinical Practice Guidelines in Oncology (NCCN Guidelines) for CNS Cancers ${ }^{29}$ currently use a 12-month interval as a cutoff; however, other factors may influence how to define a meaningful time for an individual patient (eg, adequate dosing and frequency of HD-MTX cycles). For patients who are good candidates for rechallenge with HD-MTX-based therapy, this may be a reasonable first option. ${ }^{17,30,31}$ Select chemosensitive and fit patients may then be candidates for HDC/ASCT if they have not received this therapy previously. ${ }^{32-34}$

Patients with R/R PCNSL who are not candidates for HD-MTX regimens or HDC/ASCT may be candidates for several single-agent and combination therapy regimens that have also demonstrated activity in this setting (Table 2). Mechanisms of action of the drugs used in the different published regimens encompass a broad spectrum, from chemotherapy and immunochemotherapy to antiangiogenesis and targeted strategies, as well as combinations of different strategies. Selection of the most appropriate therapy for an individual patient requires consideration of potential side effects, preference for administration route, and comfort and expertise of the treating physician. For example, treatment with ibrutinib alone or in combination with MTX and rituximab, or lenalidomide \pm rituximab may be more suitable for patients with compromised bone marrow reserve, whereas pemetrexed, which is predominantly renally cleared, will pose the same hurdles as HD-MTX for patients with significant renal impairment. Due to small patient numbers and lack of randomized trials, there is no clear evidence showing which of these strategies is most effective. Often, several treatment options appear reasonable for an individual patient. Participation in a clinical trial, if available, should therefore be strongly considered. Outside of a clinical trial, treatment with ibrutinib, lenalidomide \pm rituximab, or temozolomide is often considered a reasonable choice (Table 2) if no suitable clinical trial is available. However, responses are typically short-lived and measured in several months.

WBRT is an option for patients with multiple relapses or rapid disease progression if no better systemic therapy is available, but significant neurocognitive complications need to be carefully considered when choosing this treatment option. Given the growing portfolio of systemic options with often acceptable side effect profiles and reasonable response rates, WBRT is increasingly considered as a salvage option for patients with no systemic options. In very selected circumstances, stereotactic radiotherapy can be considered as an initial salvage therapy followed by one of the chemotherapy regimens discussed earlier..$^{35}$

\section{Novel Approaches and Targeted Therapies}

Insights into the molecular mechanisms of PCNSL pathophysiology have led to the introduction of novel agents, including targeted and immune-based therapies for the treatment of patients with R/R PCNSL. In particular, therapeutic agents targeting the B-cell receptor (BCR) and Toll-like receptor (TLR) signaling pathways with Bruton tyrosine kinase (BTK) inhibitors, PI3K/mTOR pathway with targeted agents, tumor microenvironment with immunomodulatory drugs (IMiDs), mechanisms of immune evasion with checkpoint inhibitors, and CD19 with cellular therapies have entered into phase I and II clinical trials. ${ }^{36}$

Two agents, ibrutinib (BTK inhibitor) and lenalidomide (IMiD), have data available from multiple studies and are included in the NCCN Guidelines for consideration as salvage therapies. ${ }^{29}$

A phase II multicenter study of single-agent ibrutinib at $560 \mathrm{mg} / \mathrm{d}$ orally in 52 patients with R/R PCNSL and primary vitreoretinal lymphoma (PVRL) yielded a disease control rate (including complete response, partial response, and stable disease) of $62 \%$ with a median PFS of 3.3 months in the intent-to-treat population. In patients presenting with brain and spinal cord disease at presentation (ie, excluding those with PVRL), the disease control rate was $52 \%$ and median PFS was 2 months. ${ }^{37}$ Results from phase I single-institution studies of ibrutinib at $840 \mathrm{mg} / \mathrm{d}$ orally as a single agent and in combination with chemotherapy demonstrated a higher overall response rate (ORR) at $77 \%$ to $86 \% .5,17,38$ Ibrutinib was also shown to be effective and safe when combined with rituximab and MTX.5,28 These studies also demonstrated longer duration of response compared with single-agent ibrutinib, although not in a randomized setting. Phase I and II studies of rituximab and lenalidomide in 


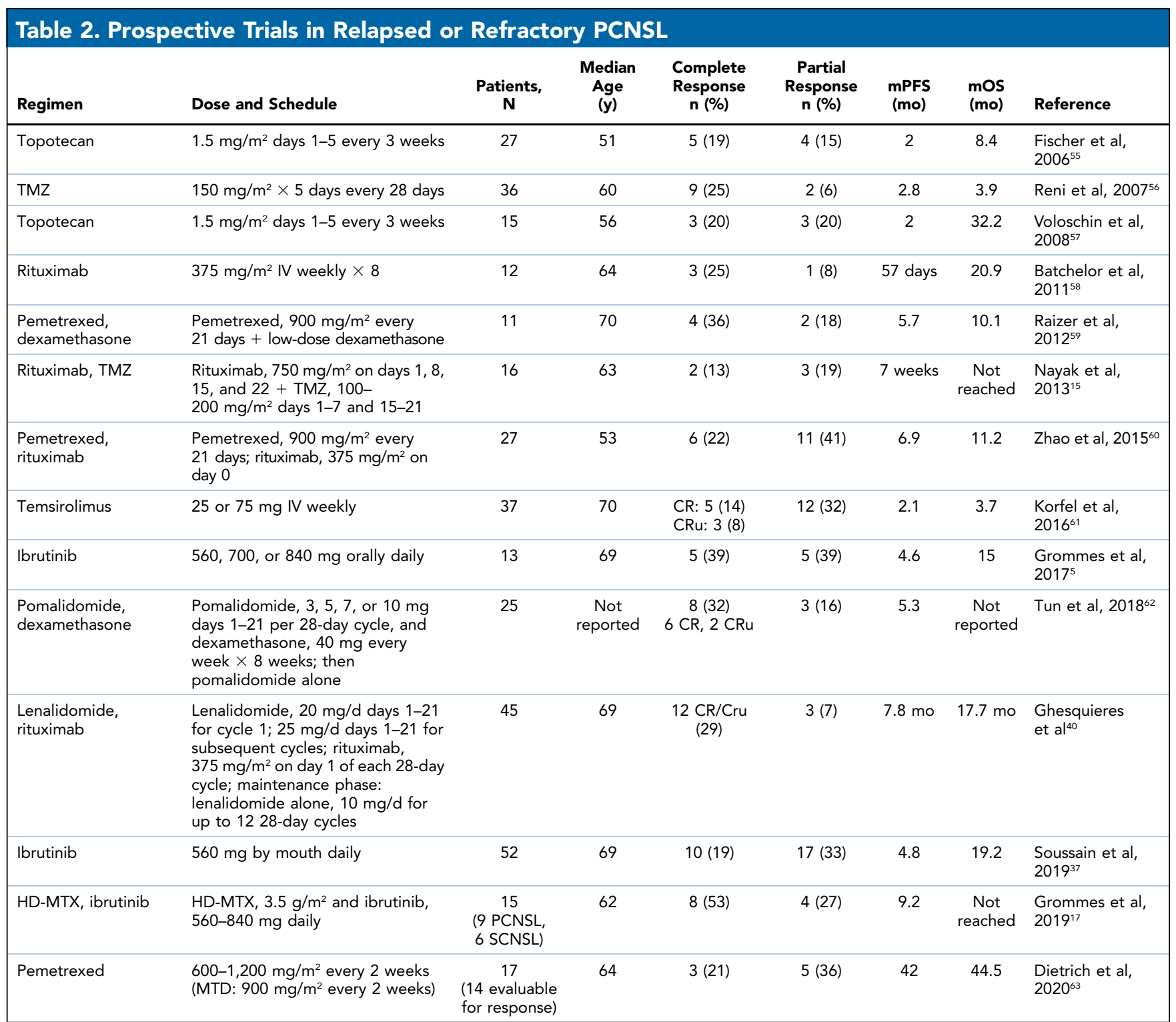

Abbreviations: $\mathrm{CR}$, complete response; CRu, complete response unconfirmed; HD-MTX, high-dose methotrexate; IV, intravenous; mOS, median overall survival; mPFS, median progression-free survival; MTD, maximum tolerated dose; PCNSL, primary central nervous system lymphoma; SCNSL, secondary central nervous system lymphoma; TMZ, temozolomide.

combination demonstrated similar results, with an ORR of $64 \%$ to $67 \%$ and median PFS of 6 to 7.8 months. ${ }^{39,40}$ Current ongoing clinical trials are investigating singleagent checkpoint inhibitors (pembrolizumab and nivolumab), various combinations of targeted agents and checkpoint inhibitors, and CD19-directed CAR T-cell therapies (ClinicalTrials.gov identifiers: NCT02779101, NCT04022980, NCT04401774). Early results from most of these studies appear promising, but caution should be exercised when considering any of these new therapies.

Despite high response rates, both ibrutinib and lenalidomide have a relatively short duration of response, and one disadvantage of using these drugs at first recurrence is that it may restrict potential trial opportunities. Both drugs are now used in combination therapy trials; moreover, newer-generation agents are also being considered, which may present better options for patients. Additionally, they may pose unique toxicities in patients with PCNSL (ie, risk of aspergillosis with ibrutinib). In general, use of novel agents is recommended in the context of clinical trial enrollment. However, in carefully selected patients, these 2 agents can be considered for patients with R/R PCNSL if clinical trials are not feasible, while closely monitoring for side effects. As such, we discourage off-label use of other novel agents in routine clinical practice for R/R PCNSL. Additionally, 
we do not recommend off-label use of any novel agents in the upfront setting or consolidation due to lack of definitive data.

\section{Borderline Candidates for HD-MTX Treatment}

Elderly patients have been classified as those older than 50 or 60 years, although most prospective studies have included patients older than 60 or 65 years. Although this age threshold remains essential when considering therapies such as WBRT, clinical trials have demonstrated that older patients do tolerate treatment with HD-MTX and that the critical factor is renal function rather than age itself. A single-institution retrospective study demonstrated that patients aged $\geq 80$ years could be successfully treated with HD-MTX. ${ }^{41}$ In general, patients with a creatinine clearance of $>40 \mathrm{~mL} / \mathrm{min}$ typically tolerate a flat dose of $3.5 \mathrm{~g} / \mathrm{m}^{2} .{ }^{12}$ Dose reduction is recommended in patients with reduced renal function and poor creatinine clearance. For HD-MTX dosing schedule of $8 \mathrm{~g} / \mathrm{m}^{2}$, the dose is reduced based on the percent reduction of the creatinine clearance below 100 $\mathrm{mL} / \mathrm{min} .{ }^{11}$ It is important to note that doses of HD-MTX as low as $1 \mathrm{~g} / \mathrm{m}^{2}$ yield brain parenchymal responses, although doses of at least $3 \mathrm{~g} / \mathrm{m}^{2}$ are recommended for adequate CSF penetration and are also associated with improved survival. ${ }^{42-44}$

\section{Challenges in Response Assessment}

PCNSL response assessment includes evaluation of brain and spinal cord with contrast MRI (contrast CT, if MRI is not possible), CSF, and eyes (detailed ophthalmologic examination), with a focus on corticosteroid use per International PCNSL Collaborative Group (IPCG) guidelines. ${ }^{45}$ We recommend making every effort to complete extent-of-disease evaluation in all patients before starting any therapy to adequately assess response, particularly when patients are neurologically stable. Although initiation of treatment in a timely fashion is important for neurologic recovery and improved survival, it is essential to avoid delays in obtaining all necessary tests. ${ }^{23}$ If significant delays are expected in patients with significant neurologic symptoms, treatment should be initiated as soon as the diagnosis is obtained, and other evaluations can occur concurrently. Every effort should be made to taper off corticosteroids once therapy is initiated to adequately assess response and prevent side effects. Minimal residual enhancement can be seen in patients who have otherwise met criteria for a complete response, which is a diagnostic challenge. These changes could be related to biopsy or focal hemorrhage and may not represent residual lymphoma. These types of responses are often classified as "complete response unconfirmed." If these changes do not change or if they slowly involute over several follow-up scans in absence of corticosteroid use, it is reasonable to consider the patient as having a complete response. ${ }^{45}$ Patients who have involvement of CSF or eyes should undergo follow-up ophthalmologic examinations to confirm response. These tests do not need to be repeated if negative at baseline as long as patients do not develop new symptoms to indicate involvement of the intraocular or subarachnoid space.

One of the major challenges with therapy for PCNSL has been development of posttreatment neurocognitive decline. This risk is higher in patients aged $>60$ years as well as long-term survivors. ${ }^{46}$ Many recent clinical trials have incorporated formal neuropsychiatric testing or the IPCG cognitive battery to follow patients; however, this is not routinely practiced in the general outpatient clinic setting. ${ }^{47}$ Comprehensive neuropsychiatric evaluation may be considered if feasible and available to follow routine patients after upfront treatment. At a minimum, the Mini Mental Status Examination (MMSE) should be performed serially during follow-up of patients, as indicated in IPCG guidelines.

\section{Conclusions}

PCNSL is in most cases a highly treatable disease, with many patients achieving long-term remission and possibly cure. Patients with appropriate renal function, independent of chronologic age and performance status and if no other contraindications are present, should be considered for HD-MTX-based therapy with curative intent. Consolidation therapy can be considered for fit patients who are well enough to tolerate HDC and/or ASCT. For patients with R/R PCNSL, HD-MTX-based therapies are often the most reasonable first option to try to reachieve a complete response. If systemic treatment options are available, WBRT should be avoided due to concerns about limited long-term control and significant neurocognitive complications. Enrollment in clinical trials should be considered in all treatment settings, as currently no uniform consensus exists regarding a standard of care, which is especially the case in R/R PCNSL and in patients not eligible for HD-MTX therapy.

Submitted August 30, 2020; accepted for publication October 7, 2020.

Disclosures: The authors have disclosed that they have no financial interests, arrangements, or affiliations with the manufacturers of any products discussed in this article or their competitors.

Funding: Dr. Nayak is supported by the Leukemia and Lymphoma Society Scholar in Clinical Research Award (LLS Grant ID: 2322-19).

Correspondence: Matthias Holdhoff, MD, PhD, The Sidney Kimmel Comprehensive Cancer Center at Johns Hopkins, 201 North Broadway, 9th Floor, Mailbox 3, Baltimore, MD 21287. Email: mholdho1@jhmi.edu; and Lakshmi Nayak, MD, Center for CNS Lymphoma, Dana Farber Cancer Institute, 450 Brookline Avenue, Boston, MA 02215.

Email: Lakshmi_Nayak@dfci.harvard.edu 


\section{References}

1. Fukumura K, Kawazu M, Kojima S, et al. Genomic characterization of primary central nervous system lymphoma. Acta Neuropathol 2016;131: 865-875.

2. Braggio $E$, Van Wier S, Ojha J, et al. Genome-wide analysis uncovers novel recurrent alterations in primary central nervous system lymphomas. Clin Cancer Res 2015;21:3986-3994.

3. Bruno A, Boisselier B, Labreche K, et al. Mutational analysis of primary central nervous system lymphoma. Oncotarget 2014;5: 5065-5075.

4. Chapuy B, Roemer MG, Stewart C, et al. Targetable genetic features of primary testicular and primary central nervous system lymphomas. Blood 2016;127:869-881.

5. Grommes C, Pastore A, Palaskas N, et al. Ibrutinib unmasks critical role of Bruton tyrosine kinase in primary CNS lymphoma. Cancer Discov 2017;7: 1018-1029.

6. Ostrom QT, Gittleman H, Truitt G, et al.CBTRUS statistical report: primary brain and other central nervous system tumors diagnosed in the United States in 2011-2015. Neuro Oncol 2018;20(Suppl):iv1-86.

7. Villano JL, Koshy $M$, Shaikh $\mathrm{H}$, et al. Age, gender, and racial differences in incidence and survival in primary CNS lymphoma. Br J Cancer 2011;105: 1414-1418.

8. Grommes C, DeAngelis LM. Primary CNS Lymphoma. J Clin Oncol 2017; 35:2410-2418.

9. Kasenda B, Ferreri AJ, Marturano E, et al. First-line treatment and outcome of elderly patients with primary central nervous system lymphoma (PCNSL) - a systematic review and individual patient data meta-analysis. Ann Oncol 2015;26:1305-1313

10. Fox CP, Phillips EH, Smith J, et al. Guidelines for the diagnosis and management of primary central nervous system diffuse large B-cell lymphoma. Br J Haematol 2019;184:348-363.

11. Batchelor T, Carson K, O'Neill A, et al. Treatment of primary CNS lymphoma with methotrexate and deferred radiotherapy: a report of NABTT 96-07. J Clin Oncol 2003;21:1044-1049.

12. Omuro A, Chinot $\mathrm{O}$, Taillandier $\mathrm{L}$, et al. Methotrexate and temozolomide versus methotrexate, procarbazine, vincristine, and cytarabine for primary CNS lymphoma in an elderly population: an intergroup ANOCEFGOELAMS randomised phase 2 trial. Lancet Haematol 2015;2:e251-259.

13. Houillier C, Taillandier L, Dureau S, et al. Radiotherapy or autologous stem-cell transplantation for primary CNS lymphoma in patients 60 years of age and younger: results of the Intergroup ANOCEF-GOELAMS randomized phase II PRECIS study. J Clin Oncol 2019;37:823-833.

14. Omuro AM, Taillandier $L$, Chinot $O$, et al. Temozolomide and methotrexate for primary central nervous system lymphoma in the elderly. J Neurooncol 2007;85:207-211.

15. Nayak L, Abrey LE, Drappatz J, et al. Multicenter phase II study of rituximab and temozolomide in recurrent primary central nervous system lymphoma. Leuk Lymphoma 2013;54:58-61.

16. Kurzwelly D, Glas M, Roth $P$, et al. Primary CNS lymphoma in the elderly: temozolomide therapy and MGMT status. J Neurooncol 2010;97: 389-392.

17. Grommes C, Tang SS, Wolfe J, et al. Phase $1 \mathrm{~b}$ trial of an ibrutinib-based combination therapy in recurrent/refractory CNS lymphoma. Blood 2019; 133:436-445

18. DeAngelis LM, Yahalom J, Thaler HT, et al. Combined modality therapy for primary CNS lymphoma. J Clin Oncol 1992;10:635-643.

19. Thiel $E$, Korfel A, Martus $P$, et al. High-dose methotrexate with or without whole brain radiotherapy for primary CNS lymphoma (G-PCNSL-SG-1): a phase 3, randomised, non-inferiority trial. Lancet Oncol 2010;11: 1036-1047

20. Correa DD, Rocco-Donovan M, DeAngelis LM, et al. Prospective cognitive follow-up in primary CNS lymphoma patients treated with chemotherapy and reduced-dose radiotherapy. J Neurooncol 2009;91: 315-321.

21. Houillier $\mathrm{C}$, Ghesquières $\mathrm{H}$, Chabrot $\mathrm{C}$, et al. Rituximab, methotrexate, procarbazine, vincristine and intensified cytarabine consolidation for primary central nervous system lymphoma (PCNSL) in the elderly: a LOC network study. J Neurooncol 2017;133:315-320.

22. Ferreri AJ, Reni M, Foppoli M, et al. High-dose cytarabine plus high-dose methotrexate versus high-dose methotrexate alone in patients with primary CNS lymphoma: a randomised phase 2 trial. Lancet 2009;374: 1512-1520.
23. Rubenstein JL, Hsi ED, Johnson JL, et al. Intensive chemotherapy and immunotherapy in patients with newly diagnosed primary CNS lymphoma: CALGB 50202 (Alliance 50202). J Clin Oncol 2013;31:3061-3068.

24. Illerhaus G, Kasenda B, Ihorst G, et al. High-dose chemotherapy with autologous haemopoietic stem cell transplantation for newly diagnosed primary CNS lymphoma: a prospective, single-arm, phase 2 trial. Lancet Haematol 2016;3:e388-397

25. Omuro A, Correa DD, DeAngelis LM, et al. R-MPV followed by high-dose chemotherapy with TBC and autologous stem-cell transplant for newly diagnosed primary CNS lymphoma. Blood 2015;125:1403-1410.

26. Schorb E, Finke J, Ferreri AJ, et al. High-dose chemotherapy and autologous stem cell transplant compared with conventional chemotherapy for consolidation in newly diagnosed primary CNS lymphoma-a randomized phase III trial (MATRix). BMC Cancer 2016;16:282.

27. Ferreri AJM, Cwynarski K, Pulczynski E, et al. Whole-brain radiotherapy or autologous stem-cell transplantation as consolidation strategies after high-dose methotrexate-based chemoimmunotherapy in patients with primary CNS lymphoma: results of the second randomisation of the International Extranodal Lymphoma Study Group-32 phase 2 trial. Lancet Haematol 2017;4:e510-523.

28. Holdhoff M, Wagner-Johnston N, Roschewski M. Systemic approach to recurrent primary CNS lymphoma: perspective on current and emerging treatment strategies. OncoTargets Ther 2020;13:8323-8335.

29. Nabors LB, Portnow J, Ahluwalia M, et al. NCCN Clinical Practice Guidelines in Oncology: Central Nervous System Cancers. Version 3.2020. Accessed October 3, 2020. To view the most recent version, visit NCCN.org

30. Plotkin SR, Betensky RA, Hochberg FH, et al. Treatment of relapsed central nervous system lymphoma with high-dose methotrexate. Clin Cancer Res 2004;10:5643-5646.

31. Pentsova E, Deangelis LM, Omuro A. Methotrexate re-challenge for recurrent primary central nervous system lymphoma. J Neurooncol 2014; 117:161-165.

32. Welch MR, Sauter CS, Matasar MJ, et al. Autologous stem cell transplant in recurrent or refractory primary or secondary central nervous system lymphoma using thiotepa, busulfan and cyclophosphamide. Leuk Lymphoma 2015;56:361-367.

33. Soussain C, Hoang-Xuan K, Taillandier L, et al. Intensive chemotherapy followed by hematopoietic stem-cell rescue for refractory and recurrent primary CNS and intraocular lymphoma: Société Française de Greffe de Moëlle Osseuse-Thérapie Cellulaire. J Clin Oncol 2008;26:2512-2518.

34. Kasenda B, lhorst G, Schroers R, et al. High-dose chemotherapy with autologous haematopoietic stem cell support for relapsed or refractory primary CNS lymphoma: a prospective multicentre trial by the German Cooperative PCNSL study group. Leukemia 2017;31:2623-2629.

35. Kumar R, Laack N, Pollock BE, et al. Stereotactic radiosurgery in the treatment of recurrent CNS lymphoma. World Neurosurg 2015;84:390-397.

36. Grommes $\mathrm{C}$, Nayak L, Tun HW, et al. Introduction of novel agents in the treatment of primary CNS lymphoma. Neuro Oncol 2019;21:306-313.

37. Soussain $\mathrm{C}$, Choquet $\mathrm{S}$, Blonski $\mathrm{M}$, et al. Ibrutinib monotherapy for relapse or refractory primary CNS lymphoma and primary vitreoretinal lymphoma: final analysis of the phase II 'proof-of-concept' iLOC study by the Lymphoma Study Association (LYSA) and the French Oculo-Cerebral Lymphoma (LOC) Network. Eur J Cancer 2019;117:121-130.

38. Lionakis MS, Dunleavy K, Roschewski M, et al. Inhibition of B cell receptor signaling by ibrutinib in primary CNS lymphoma. Cancer Cell 2017;31: 833-843.e5.

39. Rubenstein JL, Geng H, Fraser EJ, et al. Phase 1 investigation of lenalidomide/rituximab plus outcomes of lenalidomide maintenance in relapsed CNS lymphoma. Blood Adv 2018;2:1595-1607.

40. Ghesquieres $\mathrm{H}$, Chevrier $\mathrm{M}$, Laadhari $\mathrm{M}$, et al. Lenalidomide in combination with intravenous rituximab (REVRI) in relapsed/refractory primary CNS lymphoma or primary intraocular lymphoma: a multicenter prospective 'proof of concept' phase II study of the French Oculo-Cerebra Lymphoma (LOC) Network and the Lymphoma Study Association (LYSA). Ann Oncol 2019;30:621-628.

41. Welch MR, Omuro A, Deangelis LM. Outcomes of the oldest patients with primary CNS lymphoma treated at Memorial Sloan-Kettering Cancer Center. Neuro Oncol 2012;14:1304-1311.

42. Hoang-Xuan K, Taillandier L, Chinot $\mathrm{O}$, et al. Chemotherapy alone as initial treatment for primary CNS lymphoma in patients older than 60 years: a multicenter phase II study (26952) of the European Organization for Research and Treatment of Cancer Brain Tumor Group. J Clin Oncol 2003:21:2726-2731. 
43. Borsi JD, Moe PJ. A comparative study on the pharmacokinetics of methotrexate in a dose range of $0.5 \mathrm{~g}$ to $33.6 \mathrm{~g} / \mathrm{m} 2$ in children with acute lymphoblastic leukemia. Cancer 1987;60:5-13.

44. Reni M, Ferreri AJ, Guha-Thakurta N, et al. Clinical relevance of consolidation radiotherapy and other main therapeutic issues in primary central nervous system lymphomas treated with upfront high-dose methotrexate. Int J Radiat Oncol Biol Phys 2001;51:419-425.

45. Abrey LE, Batchelor TT, Ferreri AJ, et al. Report of an international workshop to standardize baseline evaluation and response criteria for primary CNS lymphoma. J Clin Oncol 2005;23:5034-5043.

46. Correa DD, Braun E, Kryza-Lacombe M et al. Longitudinal cognitive assessment in patients with primary CNS lymphoma treated with induction chemotherapy followed by reduced-dose whole-brain radiotherapy or autologous stem cell transplantation. J Neurooncol 2019;144:553-562.

47. Correa DD, Maron L, Harder H, et al. Cognitive functions in primary central nervous system lymphoma: literature review and assessment guidelines. Ann Oncol 2007;18:1145-1151.

48. Zhu JJ, Gerstner ER, Engler DA, et al. High-dose methotrexate for elderly patients with primary CNS lymphoma. Neuro Oncol 2009;11:211-215.

49. Holdhoff M, Ambady P, Abdelaziz A, et al. High-dose methotrexate with or without rituximab in newly diagnosed primary CNS lymphoma. Neurology 2014;83:235-239.

50. Glass J, Won M, Schultz CJ, et al. Phase I and II study of induction chemotherapy with methotrexate, rituximab, and temozolomide, followed by whole-brain radiotherapy and postirradiation temozolomide for primary CNS lymphoma: NRG Oncology RTOG 0227. J Clin Oncol 2016;34:1620-1625.

51. Fritsch K, Kasenda B, Hader C et al. Immunochemotherapy with rituximab, methotrexate, procarbazine, and lomustine for primary CNS lymphoma (PCNSL) in the elderly. Ann Oncol 2011;22:2080-2085

52. Morris PG, Correa DD, Yahalom J, et al. Rituximab, methotrexate, procarbazine, and vincristine followed by consolidation reduced-dose wholebrain radiotherapy and cytarabine in newly diagnosed primary CNS lymphoma: final results and long-term outcome. J Clin Oncol 2013;31: 3971-3979.
53. Ferreri AJ, Cwynarski K, Pulczynski E, et al. Chemoimmunotherapy with methotrexate, cytarabine, thiotepa, and rituximab (MATRix regimen) in patients with primary CNS lymphoma: results of the first randomisation of the International Extranodal Lymphoma Study Group-32 (IELSG32) phase 2 trial. Lancet Haematol 2016;3:e217-227.

54. Bromberg JEC, Issa S, Bakunina K, et al. Rituximab in patients with primary CNS lymphoma (HOVON 105/ALLG NHL 24): a randomised, open-label, phase 3 intergroup study. Lancet Oncol 2019;20:216-228.

55. Fischer $\mathrm{L}$, Thiel $\mathrm{E}$, Klasen $\mathrm{HA}$, et al. Prospective trial on topotecan salvage therapy in primary CNS lymphoma. Ann Oncol 2006;17:1141-1145.

56. Reni M, Zaja F, Mason W, et al. Temozolomide as salvage treatment in primary brain lymphomas. Br J Cancer 2007;96:864-867.

57. Voloschin AD, Betensky R, Wen PY, et al. Topotecan as salvage therapy for relapsed or refractory primary central nervous system lymphoma. J Neurooncol 2008;86:211-215.

58. Batchelor TT, Grossman SA, Mikkelsen T, et al. Rituximab monotherapy for patients with recurrent primary CNS lymphoma. Neurology 2011;76: 929-930.

59. Raizer JJ, Rademaker A, Evens AM, et al. Pemetrexed in the treatment of relapsed/refractory primary central nervous system lymphoma. Cancer 2012;118:3743-3748.

60. Zhao HT, Chen J, Shi SB, et al. Pemetrexed plus rituximab as second-line treatment of primary central nervous system lymphoma. Med Oncol 2015 32:351.

61. Korfel A, Schlegel U, Herrlinger U, et al. Phase II trial of temsirolimus for relapsed/refractory primary CNS lymphoma. J Clin Oncol 2016;34: 1757-1763.

62. Tun HW, Johnston PB, DeAngelis LM, et al. Phase 1 study of pomalidomide and dexamethasone for relapsed/refractory primary CNS or vitreoretinal lymphoma. Blood 2018;132:2240-2248.

63. Dietrich J, Versmee L, Drappatz J, et al. Pemetrexed in recurrent or progressive central nervous system lymphoma: a phase I multicenter clinical trial. Oncologist 2020;25:747.

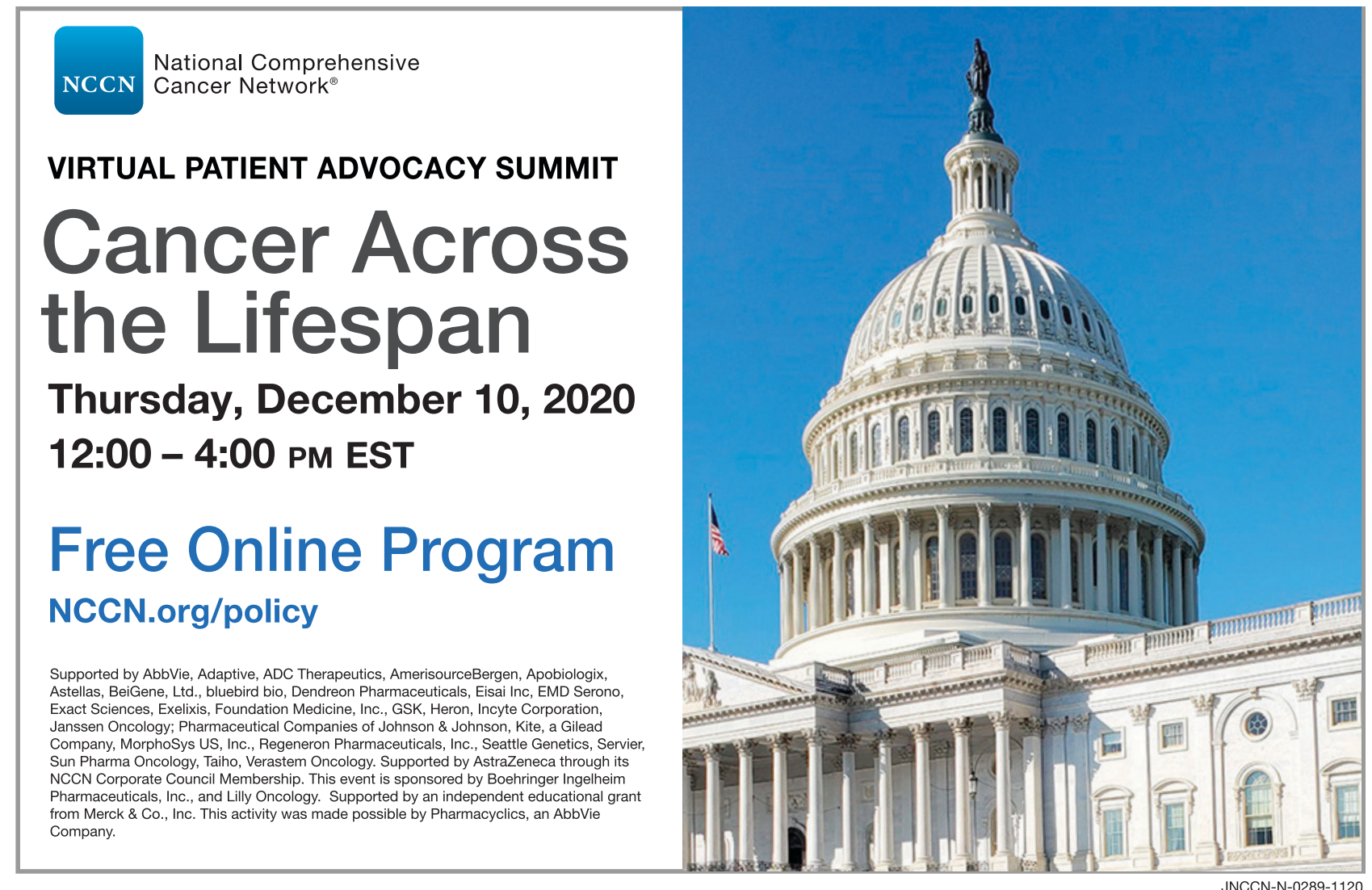

